\title{
ON GROWTH $k$-ORDER OF SOLUTIONS OF A COMPLEX HOMOGENEOUS LINEAR DIFFERENTIAL EQUATION
}

\author{
LUIS G. BERNAL
}

\begin{abstract}
In this paper, we give several results about growth $k$-order of solutions of a complex homogeneous linear differential equation with variable coefficients, provided that the coefficients are entire functions.
\end{abstract}

Denote by $C$ the complex field. Let $f: C \rightarrow C$ be an entire function. If $r>0$, we define $\exp _{1} r=\exp r, \exp _{k+1} r=\exp \left(\exp _{k} r\right)(k=1,2, \ldots)$, and $M(r)=$ $\max \{|f(z)|:|z|=r\}$. For $r>0$ large enough, we denote $\log _{1} r=\log r, \log _{k+1} r=$ $\log \left(\log _{k} r\right)(k=1,2, \ldots)$. The following definitions can be found in $[\mathbf{7}, \mathbf{8}]$. The growth $k$-order $\rho_{k}=\rho_{k}(f)$ of $f(z)$ is

$$
\rho_{k}=\limsup _{r \rightarrow \infty}\left(\log _{k+1} M(r) / \log r\right) \text {. }
$$

If $k=1$, then $\rho_{k}$ is called the order of $f(z)$, and we shall denote it by $\rho(f)$. We define the growth index $i(f)$ of $f(z)$ as $i(f)=0$ if $f(z)$ is polynomial, and $i(f)=\min \left\{k \in\{1,2, \ldots\}: \rho_{k}(f)<\infty\right\}$ if $f(z)$ is transcendental, where we set $i(f)=\infty$ when $\rho_{k}(f)=\infty$ for all $k$.

Furthermore, we shall use some notations taken from Nevanlinna theory. If $g(z)$ is a meromorphic function on $C$, one defines

$$
\begin{gathered}
m(r, g)=(1 / 2 \pi) \int_{0}^{2 \pi} \log ^{+}\left|g\left(r e^{i t}\right)\right| d t \\
N(r, g)=\int_{0}^{r}(n(t)-n(0)) / t d t+n(0) \log r
\end{gathered}
$$

and $T(r, g)=m(r, g)+N(r, g)(r>0)$, where $\log ^{+} x=\max (0, \log x)$ for $x \geq 0$ and $n(t)$ is the number of the poles of $g(z)$ lying in $|z| \leq t$, counting according to their multiplicity. $T(r, g)$ is an increasing function of $r$. If $f(z)$ is entire, then $T(r, f)=m(r, f)$ and

$$
T(r, f) \leq \log ^{+} M(r) \leq(R+r / R-r) \cdot T(R, f) \quad(0<r<R)
$$

(see [5, p. 174]). From these inequalities it is easily derived that

$$
\rho_{k}(f)=\limsup _{r \rightarrow \infty}\left(\log _{k} T(r, f) / \log r\right) .
$$

Accordingly, the $k$-order of a meromorphic function $g(z)$ is defined as $\rho_{k}(g)=$ $\lim \sup _{r \rightarrow \infty}\left(\log _{k} T(r, g) / \log r\right)$. If $k=1$, one obtains $\rho_{1}=\rho$, the order of $g(z)$.

Received by the editors June 23, 1986.

1980 Mathematics Subject Classification (1985 Revision). Primary 34A20; Secondary 30D05, $30 \mathrm{D} 15$.

Key words and phrases. Growth $k$-order, linear differential equation, entire function, Nevanlinna theory.

(C) 1987 American Mathematical Society $0002-9939 / 87 \$ 1.00+\$ .25$ per page 
Let $G \subset C$ be a simply connected domain and

$$
L(w)=0
$$

be a complex homogeneous linear differential equation of order $n$, where

$$
L(w)=w^{(n)}+a_{1}(z) w^{(n-1)}+\cdots+a_{n}(z) w
$$

and the $a_{j}(j=1, \ldots, n)$ are analytic functions on $G$, and at least one of them is not constant. We exclude the case $a_{j}=$ constant $(j=1, \ldots, n)$ because it is very well known. Each solution of (1) is analytic on $G$. In particular, if $G=C$, then each solution is entire.

In this paper, we give several bounds for the growth of solutions of (1), assuming that the functions $a_{j}$ are entire. Some particular cases can be found in [2, pp. 363366; and 4, pp. 162-169 and 340-341]. We give some sharper results when $n=2$.

We shall define the $k$-grade of the singularity of (1) as

$$
\gamma_{k}=\sup \left\{\rho_{k}(w): L(w)=0\right\} \quad(k=1,2, \ldots),
$$

and the growth index of $(1)$ as

$$
\delta=\sup \{i(w): L(w)=0\}
$$

if $G=C$.

The following lemma is known for the real case (see [3, p. 105]).

LEMMA 1. Assume that $G=I \times J \subset C$ is an open rectangle and $\left|a_{j}(z)\right|<$ $b_{j}(j=1, \ldots, n)$ for all $z \in G$. Define

$$
M=\frac{1}{2} \cdot\left(1+\max \left(2 b_{1}+b_{2}+\cdots+b_{n}, 1+b_{2}, 1+b_{3}, \ldots, 1+b_{n-1}\right)\right) .
$$

Let $z_{0}$ be a point in $G$ and $f(z)$ be a solution for (1). Let

$$
\|f(z)\|=\left(|f(z)|^{2}+\left|f^{\prime}(z)\right|^{2}+\cdots+\left|f^{(n-1)}(z)\right|^{2}\right)^{1 / 2}
$$

and $h(z)=\exp \left(M\left(\left|\operatorname{Re} z-\operatorname{Re} z_{0}\right|+\left|\operatorname{Im} z-\operatorname{Im} z_{0}\right|\right)\right)$. Then

$$
\left\|f\left(z_{0}\right)\right\| \cdot(h(z))^{-1} \leq\|f(z)\| \leq\left\|f\left(z_{0}\right)\right\| \cdot h(z)
$$

for every $z \in G$.

Proof. Let $z=x+i y \in G$ and $z_{0}=x_{0}+i y_{0}$. Then $x, x_{0} \in I$ and $y, y_{0} \in J$. We have $L(f)=0$ and $f=u+i v$, where $u, v: G \rightarrow R$. The Cauchy-Riemann equations $D_{1} u=D_{2} v$ and $D_{x} u=-D_{1} v$ hold. Since $L(f)=0$, one has $f^{(n)}=$ $-a_{1} f^{(n-1)}-\cdots-a_{n} f$, hence

$$
\left|f^{(n)}\right| \leq b_{1}\left|f^{(n-1)}\right|+\cdots+b_{n}|f|
$$

in $G$. Call $g(z)=\|f(z)\|^{2}$. Then $g=f \bar{f}+f^{\prime} \bar{f}^{\prime}+\cdots+f^{(n-1)} \overline{f^{(n-1)}}$. By partial differentiation we obtain $D_{1} g=\sum_{j=0}^{n-1}\left(\overline{f^{(j)}} \cdot D_{1} f^{(j)}+f^{(j)} \cdot D_{1} \overline{f^{(j)}}\right)$. Since each $f^{(j)}$ is analytic on $G$, we know that $D_{1} f^{(j)}=f^{(j+1)}$ and $D_{1} \overline{f^{(j)}}=D_{1}\left(D_{1}^{j} u-i D_{1}^{j} v\right)=$ $\overline{D_{1} f^{(j)}}=\overline{f^{(j+1)}}$. Then $D_{1} g=\sum_{j=0}^{n-1}\left(\overline{f^{(j)}} f^{(j+1)}+f^{(j)} \overline{f^{(j+1)}}\right)$. Thus

$$
\left|D_{1} g\right| \leq 2 \cdot \sum_{j=n}^{n-1}\left|f^{(j)}\right| \cdot\left|f^{(j+1)}\right| .
$$


By (3),

$$
\begin{aligned}
\left|D_{1} g\right| \leq & 2 \cdot \sum_{j=0}^{n-2}\left|f^{(j)}\right| \cdot\left|f^{(j+1)}\right|+2\left|f^{(n-1)}\right| \cdot \sum_{j=0}^{n-1} b_{n-j}\left|f^{(j)}\right| \\
\leq & \sum_{j=0}^{n-2}\left(\left|f^{(j)}\right|^{2}+\left|f^{(j+1)}\right|^{2}\right)+\sum_{j=0}^{n-1}\left(b_{n-j}\left|f^{(n-1)}\right|^{2}+\left|f^{(j)}\right|^{2}\right) \\
= & \left(1+b_{n}\right)|f|^{2}+\left(2+b_{n-1}\right)\left|f^{\prime}\right|^{2}+\left(2+b_{n-2}\right) \cdot\left|f^{\prime \prime}\right|^{2} \\
& +\cdots+\left(2+b_{2}\right)\left|f^{(n-2)}\right|^{2}+\left(1+2 b_{1}+b_{2}+\cdots+b_{n}\right)\left|f^{(n-1)}\right|^{2} \\
\leq & 2 M\left(|f|^{2}+\cdots+\left|f^{(n-1)}\right|^{2}\right),
\end{aligned}
$$

and so $\left|D_{1} g\right| \leq 2 M g$, or $-2 M g \leq D_{1} g \leq 2 M g$. From the right inequality, we deduce

$$
\exp (-2 M x) \cdot\left(D_{1} g(x, y)-2 M g(x, y)\right)=D_{1}(\exp (-2 M x) \cdot g(x, y)) \leq 0 .
$$

Fix $y \in J$. If $x>x_{0}$, we integrate from $x_{0}$ to $x$ obtaining $\exp (-2 M x) g(x, y)-$ $\exp \left(-2 M x_{0}\right) g\left(x_{0}, y\right) \leq 0$, whence $g(x, y) \leq g\left(x_{0}, y\right) \cdot \exp \left(2 M\left(x-x_{0}\right)\right)$. If $x<$ $x_{0}$, we integrate from $x$ to $x_{0}$ to get $\exp \left(-2 M\left(x_{0}-x\right)\right) \cdot g\left(x_{0}, y\right) \leq g(x, y)$. If we now use the left inequality $-2 M g \leq D_{1} g$ in the same way we get $g(x, y) \leq$ $\exp \left(2 M\left(x_{0}-x\right) \cdot g\left(x_{0}, y\right)\right.$ for $x<x_{0}$ and $\exp \left(-2 M\left(x-x_{0}\right)\right) \cdot g\left(x_{0}, y\right) \leq g(x, y)$ for $x_{0}<x$. We can now write this as

$$
\exp \left(-2 M\left|x-x_{0}\right|\right) \cdot g\left(x_{0}, y\right) \leq g(x, y) \leq \exp \left(2 M\left|x-x_{0}\right|\right) \cdot g\left(x_{0}, y\right) .
$$

By partial differentiation with respect to $y$ we obtain

$$
\left.D_{2} g=\sum_{j=0}^{n-1} \overline{\left(f^{(j)}\right.} \cdot D_{2} f^{(j)}+f^{(j)} \cdot D_{2} \overline{f^{(j)}}\right) .
$$

From the Cauchy-Riemann equations,

$$
\begin{aligned}
D_{2} f^{(j)} & =D_{2}\left(D_{1}^{j} u+i D_{1}^{j} v\right)=-D_{1}^{j+1} v+i D_{1}^{j+1} u \\
& =i\left(D_{1}^{j+1} u+i D_{1}^{j+1} v\right)=i f^{(j+1)}
\end{aligned}
$$

and $D_{2} f^{(j)}=-i f^{\overline{(j+1)}}$. Then (4) works equally well for $D_{2} g$. Consequently, $\left|D_{2} g\right| \leq 2 M g$, i.e., $-2 M g(x, y) \leq D_{2} g(x, y) \leq 2 M g(x, y)$. In particular

$$
-2 M g\left(x_{0}, y\right) \leq D_{2} g\left(x_{0}, y\right) \leq 2 M g\left(x_{0}, y\right)
$$

and by repeating the derivation of (5) above in $y$ instead of $x$ we obtain

$$
\exp \left(-2 M\left|y-y_{0}\right|\right) \cdot g\left(x_{0}, y_{0}\right) \leq g\left(x_{0}, y\right) \leq \exp \left(2 M\left|y-y_{0}\right|\right) \cdot g\left(x_{0}, y_{0}\right) \text {. }
$$

Combining with (5), one derives that

$$
\begin{gathered}
\exp \left(-2 M\left(\left|x-x_{0}\right|+\left|y-y_{0}\right|\right)\right) \cdot g\left(x_{0}, y_{0}\right) \leq g(x, y) \\
\leq \exp \left(2 M\left(\left|x-x_{0}\right|+\left|y-y_{0}\right|\right)\right) \cdot g\left(x_{0}, y_{0}\right)
\end{gathered}
$$

or

$$
\left\|f\left(z_{0}\right)\right\| \cdot(h(z))^{-1} \leq\|f(z)\| \leq\left\|f\left(z_{0}\right)\right\| \cdot h(z) .
$$


LEMMA 2. Let $f(z)$ be a nonconstant meromorphic function on the plane. Then

$$
m\left(r, f^{\prime} / f\right)=O(\log (r T(r, f)))
$$

as $r \rightarrow \infty$ outside an exceptional set of finite linear measure. If $f(z)$ has finite order $\rho$, then (6) holds as $r \rightarrow \infty$ through all positive real values; if $c$ is any number $c>1+3 \rho$ and $r=|z|$, then

$$
\left|f^{\prime}(z) / f(z)\right|<18 r^{c}
$$

for $r$ outside of intervals of finite total length. 123].

The first part can be found in [6] and the second part is proved in [4, pp. 121-

LEMMA 3. Let $E$ be a subset of $(0, \infty)$ with finite linear measure. If $F, G:(0, \infty)$ $\rightarrow R$ are functions satisfying

(a) $F$ is nondecreasing and $G$ is positive,

(b) $\lim _{r \rightarrow \infty} G(s(r)) / G(r)=1$ for all functions $s:(0, \infty) \rightarrow R$ such that $0<$ $s(r)-r<1(r>0)$, then

(8) $\limsup _{r \rightarrow \infty} F(r) / G(r)=\sup \left\{\limsup _{n \rightarrow \infty} F\left(r_{n}\right) / G\left(r_{n}\right): r_{1}<r_{2}<\cdots<r_{n}<\cdots\right.$, $r_{n} \rightarrow \infty$ and $\left.r_{n} \notin E(n=1,2, \ldots)\right\}$.

ProOF. We have

$$
\limsup _{r \rightarrow \infty} F(r) / G(r)=\sup \left\{\limsup _{n \rightarrow \infty} F\left(r_{n}\right) / G\left(r_{n}\right):\left\{r_{n}\right\}_{n=1}^{\infty} \in D\right\},
$$

where $D=\left\{\left\{r_{n}\right\}_{n=1}^{\infty}: r_{1}<r_{2}<\cdots\right.$ and $\left.r_{n} \rightarrow \infty\right\}$. Take $\left\{r_{n}\right\}_{n=1}^{\infty} \in D$ such that $\lim \sup _{n \rightarrow \infty} F\left(r_{n}\right) / G\left(r_{n}\right)=\limsup _{r \rightarrow \infty} F(r) / G(r)$. One can find a sequence $\left\{s_{n}\right\}_{n=1}^{\infty}$ and an integer $n_{0}$ with $s_{1}<s_{2}<\cdots<s_{n}<\cdots, s_{n} \notin E$ for every $n$, and $0<s_{n}-r_{n}<1\left(n>n_{0}\right)$, because $E$ has finite measure. Then $\left\{s_{n}\right\}_{n=1}^{\infty} \in D$ and $\lim _{n \rightarrow \infty} G\left(s_{n}\right) / G\left(r_{n}\right)=1$, so

$$
\begin{aligned}
\limsup _{n \rightarrow \infty} F\left(r_{n}\right) / G\left(r_{n}\right) & =\limsup _{n \rightarrow \infty} F\left(r_{n}\right) / G\left(s_{n}\right) \\
& \leq \limsup _{n \rightarrow \infty} F\left(s_{n}\right) / G\left(s_{n}\right) \leq \limsup _{r \rightarrow \infty} F(r) / G(r),
\end{aligned}
$$

and (8) follows.

Now, we state our result on growth. Note that part (iv) generalizes a theorem of Wittich (1948): If all solutions are of finite order, then the coefficients are polynomials. For this, see [4, pp. 167-168].

THEOREM 4. Assume that $G=C$ in (1). Let $p=\max \left\{i\left(a_{j}\right): j=1, \ldots, n\right\}$. If $p$ is finite and $p>0$, then we call $\alpha=\max \left\{\rho_{p}\left(a_{j}\right): j=1, \ldots, n\right\}$. Then the following conditions are satisfied:

(i) $\delta \leq 1+p$.

(ii) If $p$ is finite and $p>0$, then $\gamma_{p+1} \leq \alpha$.

(iii) If every $a_{j}(z)$ is a polynomial, let $m=\max \left\{\operatorname{degree}\left(a_{j}\right): j=1, \ldots, n\right\}$. Then

$$
\gamma_{1} \leq 1+m .
$$


(iv) Let $n=2$. Then $\delta=1+p$. In addition, $\gamma_{p+1}=\alpha$ if $p>0$, and $\gamma_{1} \geq$ $\frac{1}{3}\left(\operatorname{degree}\left(a_{1}\right)-1\right)$ if $p=0$.

PROOF. Let $f(z)$ be a solution for (1). Call $M(r)=\max \{|f(z)|:|z|=$ $r\}, M_{j}(r)=\max \left\{\left|a_{j}(z)\right|:|z|=r\right\}, N(r)=\sup \left\{|f(z)|: z \in G_{r}\right\}$ and $N_{j}(r)=$ $\sup \left\{\left|a_{j}(z)\right|: z \in G_{r}\right\}(j=1, \ldots, n), G_{r}$ being $G_{r}=(-r, r) \times(-r, r) \subset C$. From the inequalities $N(r / 2) \leq M(r) \leq N(2 r)$ and $N_{j}(r / 2) \leq M_{j}(r) \leq N_{j}(2 r)$, it is obvious that one can replace $M(r)$ by $N(r)$ and $M_{j}(r)$ by $N_{j}(r)$ in the respective expressions of $k$-order.

If $p=\infty$, then (i) is trivial. Otherwise, there exist real numbers $r_{0}, d_{1}, \ldots, d_{n}>0$ such that $N_{j}(r)<\exp _{p}\left(r^{d_{j}}\right)\left(j=1, \ldots, n ; r>r_{0}\right)$. Apply Lemma 1 for $z_{0}=0$ and $G_{r}$. We obtain $|f(z)| \leq\|f(z)\| \leq\|f(0)\| \cdot \exp \left(2 r+2 r N_{1}(r)+\cdots+2 r N_{n}(r)\right)$ if $z \in G_{r}$ and $r>r_{0}$, because $M \leq 1+N_{1}(r)+\cdots+N_{n}(r)$. If $d \in\left(1+\max \left(d_{1}, \ldots, d_{n}\right), \infty\right)$, one can find $r_{1}>r_{0}$ with $2 r+\cdots+2 r N_{n}(r)<\exp _{p}\left(r^{d}\right)$ when $r>r_{1}$. If $s \in(d, \infty)$, there is an $r_{2}>r_{1}$ such that $\|f(0)\| \cdot \exp \left(2 r+\cdots+2 r N_{n}(r)\right)<\exp _{p+1}\left(r^{s}\right)$ when $r>r_{2}$. Thus $N(r)<\exp _{p+1}\left(r^{s}\right)$ and $\left(\log _{p+2} N(r)\right) / \log r<s$ asymptotically (asymp.). Hence $\rho_{p+1}(f)$ is finite and $i(f) \leq 1+p$, so $\delta \leq 1+p$.

(ii) From the definition of $\alpha$, it is clear that, given $\varepsilon>0$, one has $N_{j}(r)<$ $\exp _{p}\left(r^{\alpha+\varepsilon}\right)$ for $r>r_{0}$. But it is easily derived that there is $r_{1}>r_{0}$ with $\|f(0)\|$. $\exp \left(2 r+\cdots+2 r N_{n}(r)\right)<\exp _{p+1}\left(r^{\alpha+2 \varepsilon}\right)$, whence $N(r)<\exp _{p+1}\left(r^{\alpha+2 \varepsilon}\right)$ asymp. Hence, $\left(\log _{p+2} N(r)\right) / \log r<\alpha+2 \varepsilon$ asymp. and $\rho_{p+1}(f) \leq \alpha+2 \varepsilon$ for each $\varepsilon>0$, i.e., $\rho_{p+1}(f) \leq \alpha$ and $\delta_{p+1} \leq \alpha$.

Similar arguments to those of (ii) let us prove (iii), taking into account that $2 r+2 r N_{1}(r)+\cdots+2 r N_{n}(r)<r^{m+1+\varepsilon}$ for all $r>r_{0}(\varepsilon)>0$. Hence, (9) holds.

(iv) In this case, $L(w)=w^{\prime \prime}+a_{1}(z) w^{\prime}+a_{2}(z) w$. Let $\left\{w_{1}, w_{2}\right\}$ be a fundamental system of solutions. The wronskian $W=w_{1} w_{2}^{\prime}-w_{1}^{\prime} w_{2}$ is entire and $\rho_{k}(W) \leq \max \left(\rho_{k}\left(w_{1}\right), \rho_{k}\left(w_{2}\right)\right)$, because $\max \left(\rho_{k}(g h), \rho_{k}(g+h)\right) \leq \max \left(\rho_{k}(g), \rho_{k}(h)\right)$ and $\rho_{k}\left(g^{\prime}\right)=\rho_{k}(g)$ if $g, h$ are entire (see, for instance, $[1$, pp. 28, 37, and 43]).

Then $a_{1}(z)=-W^{\prime} / W$ and $a_{2}(z)=-\left(w^{\prime \prime} / w\right)-a_{1}\left(w^{\prime} / w\right)=-\left(w^{\prime \prime} / w^{\prime}\right) \cdot\left(w^{\prime} / w\right)-$ $a_{1}\left(w^{\prime} / w\right)$ if $L(w)=0$. Furthermore, $\gamma_{k}=\max \left(\rho_{k}\left(w_{1}\right), \rho_{k}\left(w_{2}\right)\right)$, because $w=$ $a w_{1}+b w_{2}$ if $L(w)=0(a, b \in C$, depending on $w)$. From Nevanlinna theory, $m\left(r, a_{2}\right)=m\left(r, w^{\prime \prime} / w^{\prime}\right)+2 m\left(r, w^{\prime} / w\right)+m\left(r, a_{1}\right)+O(\log r)$.

If $\delta=0$, then $i(w)=0$ for all solutions $w$, that is, every solution is a polynomial. But it is well known that this is possible only if $L(w)=w^{\prime \prime}$ and this case is excluded. Then $\delta=1+p$ is trivial if $p=0$.

Let $p>0$. By contradiction, assume $0<\delta<1+p$. then $\delta \leq p$ and $i(w) \leq p$ if $L(w)=0$. Hence $\rho_{p}\left(w_{j}\right)$ is finite $(j=1,2)$ so $\rho_{p}(W)$ is finite. Then $m\left(r, a_{1}\right)=$ $m\left(r, W^{\prime} / W\right)=O(\log (r \cdot T(r, W)))$ outside $E$ with finite measure by Lemma 2 . We have

$$
\log _{p-1} m\left(r, a_{1}\right)=O\left(\log _{p}(r \cdot T(r, W))\right)=O\left(\log _{p} r+\log _{p} T(r, W)\right)=O(\log r)
$$

outside $E$, because $\log _{p} T(r, W)=O(\log r)$. By using Lemma 3, with $F(r)=$ $\log _{p-1} m\left(r, a_{1}\right)$ and $G(r)=\log r$, we conclude that $\rho_{p-1}\left(a_{1}\right)$ is finite if $p>1$, and $a_{1}$ is a polynomial if $p=1$. But, again from Lemma 2 , outside a set $E$ as above,

$$
\begin{aligned}
m\left(r, a_{2}\right)= & O(\log (r T(r, w)))+O\left(\log \left(r T\left(r, w^{\prime}\right)\right)\right) \\
& +O\left(\log \left(r T\left(r, a_{1}\right)\right)\right)+O(\log r) .
\end{aligned}
$$


Since $\rho_{p}(w)=\rho_{p}\left(w^{\prime}\right)$, we reason as above that $\rho_{p-1}\left(a_{2}\right)$ is finite if $p>1$ or $a_{2}$ is a polynomial if $p=1$. Thus $p=\max \left(i\left(a_{1}\right), i\left(a_{2}\right)\right) \leq p-1$, a contradiction. Then $\delta=1+p$.

If $p>0$, assume $\gamma_{p+1}<\alpha$. Then $\alpha>0, \rho_{p+1}(w)<c$ if $L(w)=0$, and $\rho_{p+1}(W)<c$ for some $c \in(0, \alpha)$. Given $\varepsilon>0, \log _{p+1} T(r, W)<(c+\varepsilon) \log r$ asymp. for every $\varepsilon>0$. Then

$$
\log _{p} m\left(r, a_{1}\right)=\log _{p}(O(\log (r T(r, W))))<(c+2 \varepsilon) \log r
$$

outside $E=E(\varepsilon)$ with finite measure. From Lemma 3,

$$
\limsup _{r \rightarrow \infty}\left(\log _{p} m(r, a) / \log r\right)<c+2 \varepsilon
$$

for every $\varepsilon>0$, i.e., $\rho_{p}\left(a_{1}\right) \leq c$. From (10), one obtains similarly $\rho_{p}\left(a_{2}\right) \leq c$. Hence $\alpha=\max \left(\rho_{p}\left(a_{1}\right), \rho_{p}\left(a_{2}\right)\right)<\alpha$, a contradiction, so $\gamma_{p+1}=\alpha$.

If $p=0$, assume $\gamma_{1}<L$, where $L=\frac{1}{3}\left(\operatorname{degree}\left(a_{1}\right)-1\right)$. then $\operatorname{degree}\left(a_{1}\right)>$ $1, \rho(w)<d$ if $L(w)=0$, and $\rho(W)<d$ for some $d \in(0, L)$. Let $c>1+3 \rho(W)$. By Lemma 2 and (7), we have

$$
m\left(r, a_{1}\right)=m\left(r, W^{\prime} / W\right)=(1 / 2 \pi) \cdot \int_{0}^{2 \pi} \log ^{+}\left|W^{\prime}\left(r e^{i t}\right) / W\left(r e^{i t}\right)\right| d t<c \cdot \log r
$$

for $r$ outside $E=E(c)$ of finite linear measure. By Lemma 3,

$$
\limsup _{r \rightarrow \infty} m\left(r, a_{1}\right) / \log r \leq c,
$$

whence $\lim \sup _{r \rightarrow \infty} m\left(r, a_{1}\right) / \log r \leq 1+3 \rho(W)$. From Nevanlinna theory,

$$
\operatorname{degree}\left(a_{1}\right) \leq 1+3 \rho(W)<1+3 d<1+3 L=\operatorname{degree}\left(a_{1}\right)
$$

a contradiction. Thus $\gamma_{1} \geq L$. The theorem is proved.

\section{REFERENCES}

1. L. Bernal, Tesis, Facultad de Matemáticas, Universidad de Sevilla, Spain, 1984.

2. L. Bieberbach, Theorie der gewöhnlichen differentialgleichungen, Springer-Verlag, Berlin and New York, 1965.

3. E. A. Coddington, An introduction to ordinary differential equations, 5th printing, PrenticeHall, Englewood Cliffs, N.J. 1964.

4. E. Hille, Ordinary differential equations in the complex domain, Wiley, New York, 1984.

5. A. S. B. Holland, Introduction to the theory of entire functions, Academic Press, New York and London, 1973.

6. R. Nevanlinna, Analytic functions, Springer-Verlag, Berlin and New York, 1970.

7. A. R. Reddy, On entire Dirichlet series of infinite order, Rev. Mat. Hisp.-Amer. 27 (1967), 120-131.

8. D. Sato, On the rate of growth of entire functions of fast growth, Bull. Amer. Math. Soc. 69 (1963), 410-414.

Departamento de TeOría de Funciones, FaCultad de Matemáticas, C./TARFia S.N., SEVILla 41012, SPAIN 